\title{
A developmental analysis of brightness discrimination learning in the rat: Evidence for an attentional deficit
}

\author{
JERRY W. RUDY and CARL ANDREW CASTRO \\ University of Colorado, Boulder, Colorado
}

\begin{abstract}
The ability of 16-28-day-old Long-Evans rats to solve simultaneous and successive brightness discrimination problems was assessed in a novel water-maze version of the Lashley jump stand. Performance on the two problems was virtually identical at every age, but older pups mastered both problems in far fewer trials than did younger pups (Experiment 1). Subsequent experiments, based on a two-stage attention theory analysis of discrimination learning, pointed to an attentional deficit underlying the relatively poor performance of the young rats.
\end{abstract}

Although the 17-day-old rat is capable of being conditioned to a visual stimulus (Moye \& Rudy, 1985), recent research suggests that the processes that enable it to learn other behaviors mediated by its visual system continue to develop (Rudy, Stadler-Morris, \& Albert, 1987). Using the Morris water maze (Morris, 1981), Rudy et al. (1987) studied the development of two forms of spatial navigation that are also mediated by the rat's visual system. In one case, rats could navigate to an escape platform simply by using the visible proximal cues that were co-occurrent with it. In another case, the platform was hidden beneath the water's surface, but the rat could navigate to it by learning the platform's location relative to extramaze distal cues. Adult rats easily solve both problems (Morris, 1981). Rudy et al. (1987), however, found that these two forms of navigation were dissociated during ontogenesis: Rats only 17 days old were able to solve the proximal-cue problem, but it was not until the pups were 20 days old that the first evidence of distalcue navigation was observed.

The purpose of the present research was to further investigate the development of learned behaviors mediated by the visual system by studying the development of brightness discrimination learning. Since Lashley (1929/1963) and Krechevsky (1932), many theorists have entertained what is called a two-stage theory of discrimination learning (e.g., Lawrence, 1949; Mackintosh, 1965; Sutherland \& Mackintosh, 1971). In the first stage, the animal must learn to attend to the stimulus dimension that is relevant to the solution of the problem (e.g., brightness). In the second stage, the animal comes to associate the specific values of that dimension (e.g., black or white) with the correct and incorrect choice outcomes.

This research was funded in part by NSF Grant BNS-8207654 and NIH Grant DA03531 awarded to Jerry W. Rudy. We thank Peter Albert for his assistance in the collection of the data, and Tom Moye for his valuable comments on earlier drafts of this manuscript. Requests for reprints should be sent to Jerry W. Rudy, Department of Psychology, University of Colorado, Boulder, CO 80309.
Within this framework, the discrimination performance of young animals might be impaired relative to that of older animals because of maturational differences in either their associative or attentional processes. By systematically analyzing brightness discrimination learning by different-aged subjects, our goal was to delineate differences in attentional and associative processes that might exist during the period of ontogenesis in which the capac-

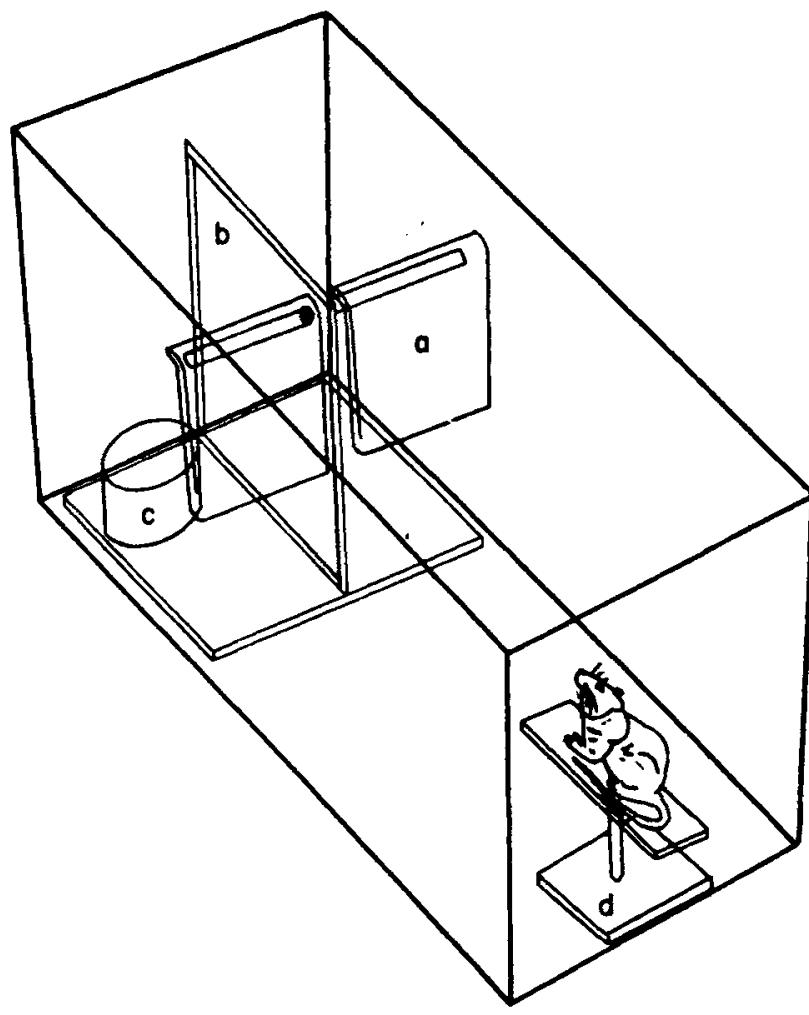

Figure 1. A schematic drawing of the apparatus used to study both the simultaneous and successive brightness discrimination problems: $a=$ the plastic stimulus card holders, $b=$ the clear plastic dividing partition; $c=$ the excape platform; and $d=$ the observation platform. 
ity to solve brightness discrimination problems is emerging. In these experiments, we compared the performance of different-aged rats on two visual discrimination learning tasks. One was a simultaneous brightness discrimination, and the other was a successive conditional brightness discrimination (Lawrence, 1949).

To study the performance of young rats on both of these problems, we developed a new methodology that combined the virtues of the Morris (1981) water maze with those of the Lashley (1930) jump stand. Our previous experience using the Morris water maze (Rudy et al., 1987; see also Schenk, 1985) had indicated that young rats adapt readily to water but are highly motivated to escape by swimming to a safe platform.

In our task (see Figure 1), the pups were placed on an observation platform facing the discriminanda, but rather than being required to jump to the correct stimulus, they were placed in the water and required to swim under the stimulus card to find the escape platform. On the simultaneous problem, the subjects were presented with two stimulus cards, one black and one white, and the platform was always behind either the black or the white card. On the successive conditional problem, the subjects were also presented with two cards, but, on a given trial, the cards were either both black or both white. The location of the platform was contingent upon whether the cards were both black or both white. For example, if both were white, the platform was behind the card on the right, and if both were black, it was behind the card on the left.

\section{EXPERIMENT 1}

A comparison of how different-aged rats perform on simultaneous and successive discrimination problems is of interest for several reasons. First, no systematic analysis of how the rat's performance changes during development is available for either task. Second, a developmental analysis can potentially be useful in determining the degree to which the performance of these two problems depends upon the same underlying processes. Evidence for different underlying processes would take the form of a developmental dissociation in which the age at which rats were able to solve one problem was different from the age at which they could solve the other. Thus, in Experiment 1 , we assessed the ability of pups $16,18,20$, and 28 days of age to learn both simultaneous and successive conditional brightness discriminations.

\section{Method}

Subjects. The subjects were Long-Evans-derived hooded rat pups of both sexes bred at the University of Colorado. Pregnant female rats were checked for births each day between 9:00 and 11:00 a.m. A new litter was considered to be 0 days old. Each litter was culled to 9 pups 2-3 days after birth, and housed with the dam in a $45 \times 25 \times 20 \mathrm{~cm}$ Plexiglas maternity cage containing pine shavings. The dams had continuous access to Wayne laboratory chow and water. When the pups were 21 days old, the dam was removed from the cage. The subjects were in a colony with a 12:12 light/dark cycle.
Apparatus. The apparatus (see Figure 1) consisted of an aquarium that contained two stimulus card holders, a partition, an escape platform, and an observation platform. The discriminanda (the black or white stimulus cards) were inserted into clear plastic stimulus card holders $(19 \mathrm{~L} \times 14 \mathrm{~W} \mathrm{~cm})$ that were suspended from the partition. The partition, a clear Plexiglas panel, was fitted with suspending rods so that the bottoms of the stimulus card holders were $2 \mathrm{~cm}$ above the water level and $40 \mathrm{~cm}$ from the back wall of the aquarium.

The 9-cm-diam escape platform was located on either one or the other side of the partition, and behind the stimulus card holder. The top of the platform was $2.0 \mathrm{~cm}$ below the surface of the water. The observation platform $(16 \mathrm{~L} \times 6 \mathrm{~W} \mathrm{~cm})$, located at the opposite end of the aquarium, protruded $6.5 \mathrm{~cm}$ above the water surface. The distance between the forward end of the observation platform and the discriminanda was $24 \mathrm{~cm}$.

The exterior of the glass aquarium $(60 \mathrm{~L} \times 30 \mathrm{~W} \times 39 \mathrm{H} \mathrm{cm})$ tank was covered by brown paper. An opaque top covered the end of the aquarium where the escape platform was located. The water was $10.5 \mathrm{~cm}$ deep and was rendered opaque by white Crayola powder paint ( $20 \mathrm{~g} / 19.8 \mathrm{liter}$ ). The water temperature was maintained at $30^{\circ}, 28^{\circ}, 26^{\circ}$, and $24^{\circ} \mathrm{C}$ for $16,17-18,19-20$, and $21-28$-dayold pups, respectively. The tank was cleaned and the water was changed daily.

Design and subject assignment. At each age, two litters each contributed 4 subjects to both the simultaneous and successive conditional training conditions ( $n=8$ at each age). Males and females were distributed as equally as possible among the various conditions. Different groups of pups began training at $16,18,20$, and 28 days of age. The average weights of the pups for the different age groups were $28.5,30.4,33.3$, and $71.1 \mathrm{~g}$ for the 16-, 18-, 20-, and 28-day-olds, respectively. For half of the subjects trained on the simultaneous problem, the escape platform was always located behind the black card; for the other half, it was behind the white card. For half the subjects trained on the conditional problem, the platform was on the right when both cards were black and on the left when both cards were white; the opposite was true for the other half. Since performance on the two problems was independent of which stimulus card was correct or whether the platform was on the left or right when the two cards were black or white, the counterbalancing factor will be ignored in the data presentation.

Pretraining. Prior to each training session, subjects were given two warm-up trials with the stimulus cards and holders absent. On each trial, the pup was placed on the observation platform facing the direction of the escape platform. Ten seconds later it was removed and placed in the water 8 to $10 \mathrm{~cm}$ from the escape platform and allowed to swim onto it. The escape platform was located once on each side of the partition.

Training. After the last pretraining trial, the pup was placed back on the observation platform facing the back wall of the aquarium. The appropriate stimulus cards were then put in place and the pup was turned to face the stimulus cards for $15 \mathrm{sec}$. If the pup turned and faced away from the cards, the experimenter gently repositioned it. The pup and observation platform were then removed from the apparatus. The pup was then placed in the water facing the center of the back wall of the maze. A trial ended when the pup swam under the stimulus card holder on the side of the partition that contained the escape platform. It was then allowed to sit on the escape platform for $20 \mathrm{sec}$. If the pup made an incorrect choice, it was required to correct the error. The next trial was again begun by placement of the pup on the observation platform in the apparatus. At all times, the experimenter stood at the back of the aquarium facing the discriminanda.

A training session consisted of 26 trials. For the subjects in the simultaneous black versus white discrimination groups, each stimulus card appeared equally often on each side. The schedule of al- 
ternation required that the same stimulus card (e.g., black) could never be on one side for more than 3 consecutive trials and that transitions could occur after 1, 2, or 3 trials. This yielded a total of 14 transition trials in which the location of the stimulus cards was reversed, 7 occurring in each of the first and second blocks of 13 trials. Since the escape platform's location was always behind either the black (B+ vs. $\mathrm{W}-$ ) or the white $(B-v s . \mathrm{W}+$ ) stimulus card, it was equally often on the right and left sides of the dividing partition.

For half the trials in the successive conditional discrimination problem, both stimuli were either black or white. In all other respects the alternating schedules were identical in the two problems.

Dependent variables. The major dependent variables were: (1) trials to criterion, with the pup being said to have learned the task when it had swum to the positive stimulus in 7 of 8 trials in a single session, and (2) percentage of correct responses, as denoted by the percentage of trials on which the pup swam first to the positive stimulus.

\section{Results}

The major findings of this experiment (see Figure 2) can be summarized easily. First, performance on both the simultaneous and successive problems was age-dependent: older rats mastered both problems faster than did the younger rats. Second, performance on the two discrimination problems was virtually identical at every age.

An age $\times$ task analysis of variance on the trials to criterion measure revealed a significant effect of age $[F(1,56)$ $=273.53, p<.01$ ], but neither the task main effect nor the age $\times$ task interaction approached significance. Post hoc comparisons revealed that pups that started training when they were 16 or 18 days old took more trials to reach criterion than did pups that started training when they were 20 or 28 days old. Also, the 20 -day-olds took more trials than did the 28-day-olds (Newman-Keuls, $p<.01$ ).

\section{Discussion}

A critical task for developmental analysis is to determine why the discrimination performance of younger pups

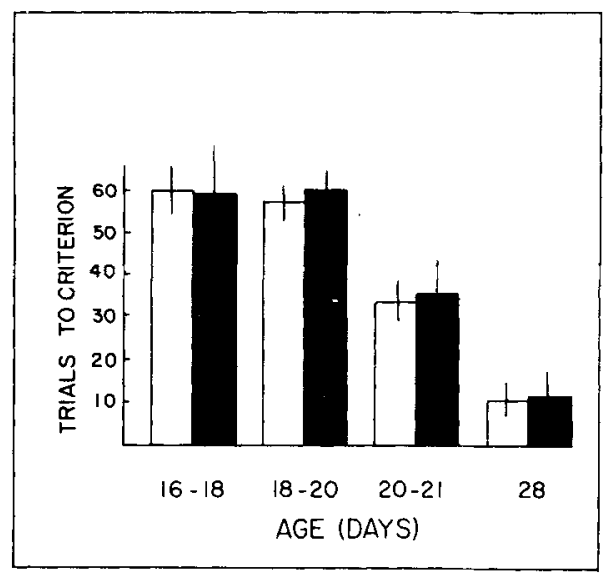

Figure 2. Mean number of trials to criterion for both the simultaneous and successive brightness problems as a function of age. Solid bars represent scores on the successive problem, and open hars represent scores on the simultaneous problem (Experiment 1). is impaired relative to that of older pups. As noted, within a two-stage theoretical analysis, the impaired discrimination performance of younger relative to older animals could reflect maturational differences in either their associative or their attentional processes.

The historical starting point for dissociating the contributions of attentional and associative processes to discrimination learning was Lashley's (1929/1963) observations about the performance of individual subjects that were learning a simultaneous brightness discrimination. He noticed that performance usually changed abruptly from chance to virtually perfect.

The stationary period during which performance is at chance is often referred to as the "presolution" period. According to two-stage theory, this reflects the period when the subject's performance is under the control of an irrelevant stimulus dimension. The abrupt change to near-perfect performance is interpreted as marking the moment when the subject has begun to attend to the relevant stimulus dimension, allowing it to quickly learn to associate the specific stimulus values with the trial outcomes.

Given this perspective, one can ask if the age-related differences in discrimination learning reflect maturational differences in the attentional processes or those in the associative processes.

The data presented in Figure 3 provide a basis on which this judgment can begin to be made. It presents "backward learning curves" (see Sutherland \& Mackintosh, 1971) for the different-aged subjects trained in Experiment 1 . This treatment of the data adjusts for the fact that individual subjects at each age reached criterion following different numbers of "presolution" trials. To obtain these curves, we identified the block of 5 trials in which each subject finished its run to criterion. The mean percentage of correct responses on this block of trials is the terminal point of the curves in Figure 3 (labeled 0 on the abscissa). Working backward from this point, the remaining data points are the mean percentage of correct responses for each block of 5 trials preceding criterion completion. In this figure, we collapsed across the simultaneous and successive problems so that 16 subjects at each age contributed to the data.

Viewed this way, one can see that the primary difference between younger and older pups was the duration of the "presolution" period, which was much longer for the younger pups than for the older pups. The rate of transition from the presolution to the solution period, however, was equally rapid for pups at all ages.

Within the context of two-stage theory, the transition data would mean that there is little or no ontogenetic Stage 2 difference in the rate at which the association between the correct stimulus and the escape platform is formed. The younger pups' relatively prolonged presolution period could then mean that the primary difference between younger and older pups lies in the Stage 1 attentional processes. 


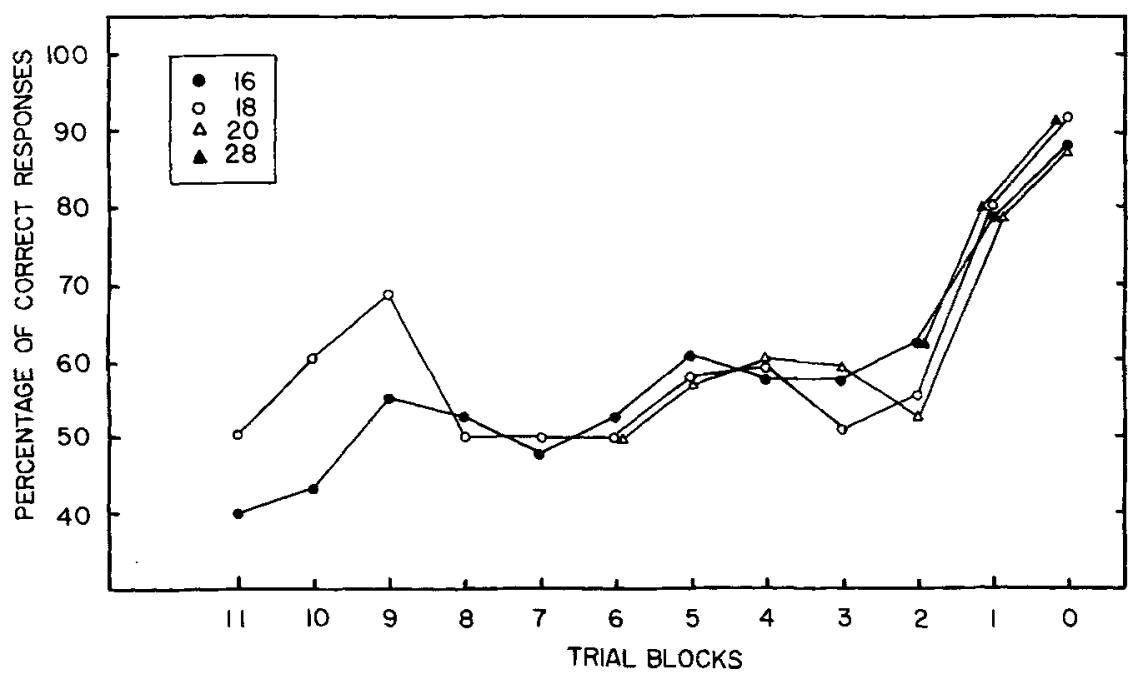

Figure 3. "Backward" learning curves for the different-aged pups in Experiment 1. See text for a complete description of how these data were obtained.

\section{EXPERIMENT 2}

Experiment 2 was designed to evaluate the attentional explanation. Recall that the presolution period for animals that began training when they were 16 days old lasted (see Figure 3) at least two sessions (52 trials). In Experiment 2, 16-day-olds received two training sessions on either the simultaneous or the conditional brightness problems. In the third session (the session during which pups this age had reached criterion in Experiment 1), we reversed the significance of the stimuli for half of the pups trained on each problem. For example, if the simultaneous problem had been $B+/ W$ - in the first two sessions, it became $\mathrm{B}-/ \mathrm{W}+$ in the third session, and if the conditional problem had been $B$, go right/W, go left, it was reversed to $B$, go left/W, go right. The contingencies operating during the first two sessions remained in effect during the third session for the remaining subjects.

If the attentional interpretation is correct, and the presolution period is prolonged because the pups are not attending to the brightness cues and consequently do not learn about their significance, then the pups in the reversal condition should reach criterion as fast on Day 3 of training as the pups in the nonreversal condition. On the other hand, however, it is possible that the younger pups were learning about the contingencies associated with the specific brightness cues but that the strength of these associations was not sufficient to influence the pups' performance (see Spence, 1936). If the latter hypothesis is correct, then pups in the reversal condition should take more trials to reach criterion than the pups in the nonreversal condition, because what they had learned (e.g., $\mathrm{B}+/ \mathrm{W}-)$ would interfere with what they must now learn to reach criterion in the third session $(\mathrm{B}-/ \mathrm{W}+)$. This negative transfer strategy has been used many times to address the same issue in the adult discrimination learning litera- ture (see Ehrenfreund, 1948; Krechevsky, 1938; Spence, 1945).

\section{Method}

Four litters each contributed 8 subjects to this experiment. All subjects were 16 days old at the start of training. Half the subjects in each litter were assigned to the simultaneous brightness discrimination problem $(n=16)$ and half to the successive conditional brightness discrimination problem $(n=16)$.

Phase 1 of training began when the pups were 16 days old and consisted of two 26-trial sessions administered on successive days. Phase 2 was initiated on the third day of training. For half the subjects trained on each problem, the significance of the stimuli was reversed (R); for the remaining half, the significance was not reversed (NR). Within each problem subset, 4 subjects from each litter were assigned to the reversal condition and 4 were assigned to the nonreversal condition ( $n=8$ for each of the four final conditions). In all other respects, the procedures employed were identical to those used in Experiment 1.

\section{Results and Discussion}

The first point to note is that no subject in either the simultaneous or the conditional training condition reached criterion during the 52 trials of Phase 1 , thus replicating the results of the first experiment. More importantly, however, pups in the reversal conditions reached criterion as quickly as did the nonreversal pups (see Figure 4). This was true for both the simultaneous and the conditional problems. This finding suggests that pups learned little or nothing about the significance of the visual cues during Phase 1 , and therefore supports the attentional deficit hypothesis.

\section{EXPERIMENT 3}

The results of Experiment 2 provide no evidence that, during the presolution period, 16-17-day-olds learned about the contingencies associated with the black and 


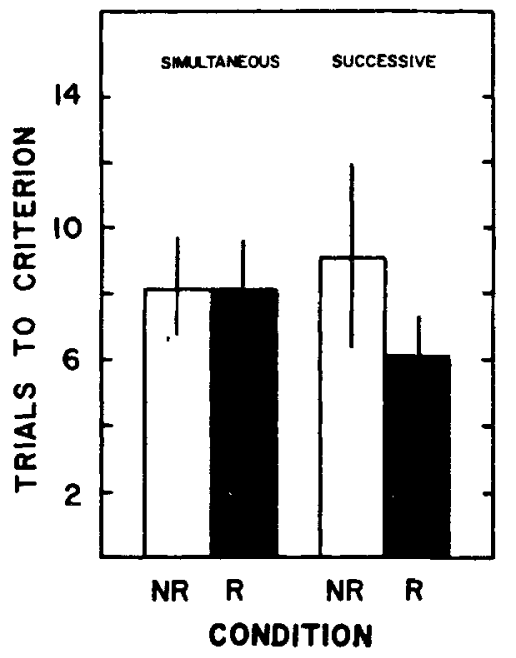

Figure 4. Mean number of trials to criterion on the simultaneous and successive problems during the third training session of Experiment 2. Key: $N R=$ nonreversal, $R=$ reversal.

white discriminanda. Consider, however, an observation that can be derived from Experiment 1. Specifically, even though pups trained when 16 and 17 days old did not reach criterion during their first two training sessions, they did reach criterion when they were 18 days old. Thus, their performance at 18 days of age was far superior to that of pups that did not begin training until they were 18 days old. The latter pups did not reach criterion until they were 20 days old. If, as indicated by the results of Experiment 2, the 16-17-day-olds failed to learn about the specific contingencies during the presolution period, what had they learned that facilitated their subsequent performance when they were 18 days old, as opposed to pups that were experimentally naive on Day 18 ?

Another possibility can be derived from two-stage attentional theory. Perhaps the pups had just begun to attend to the relevant stimulus dimension (brightness), and perhaps it was this learning that facilitated their performance on Day 18, making for the difference found between them and the naive pups.

In addition to such a specific source of transfer, however, there is a great deal of nonspecific learning that could have facilitated performance of the 18-day-olds that had been trained when they were 16 and 17 days old. For example, they had the opportunity to habituate to being in the water, to learn that there was an escape platform, and to learn how to climb onto it. It is likely that the opportunity to become generally familiar with the training environment could contribute to subsequent performance. Experiment 3 was designed to compare the contribution of these specific and nonspecific sources of transfer to the 18-day-old pups' performance.

\section{Method}

Two litters each contributed 8 pups to this experiment, 4 to each of two training conditions. The pups were 16 days old at the start of training. One group $(n=8)$ was trained on the simultaneous brightness problem in the same manner as in the previous experiments. One subject in this group was lost due to procedural error, reducing the $n$ to 7. Pups in the procedural control group $(n=8)$ were treated exactly like the pups trained on the simultaneous problem with one major difference. During their first two sessions, the black and white stimulus cards were not inserted into the clear plastic card holders. On each trial, these pups had to locate the invisible platform, which alternated from side to side according to the schedule used on the simultaneous problem. This ensured that pups in the control group had the opportunity to become familiar with the general requirements of the task, but gave them no opportunity to learn that brightness was the relevant dimension. It also meant that there were no relevant cues available. On the third day of training, when the pups were 18 days old, the black and white stimulus cards were inserted into plastic card holders and the control pups were challenged to solve the simultaneous brightness discrimination.

\section{Results}

No subjects trained initially on the simultaneous problem reached criterion during the 52 trials of training administered during the first two sessions. Note, however, that in the third session, these pups reached criterion in many fewer trials (see Figure 5) than did the procedural control pups that were transferred to the simultaneous problem $[t(13)=3.10, p<.01]$.

Even though 16-17-day-olds displayed no evidence of learning the contingencies associated with the black and white discriminanda (Experiment 2), they took only about a third as many trials to reach criterion as did the procedural controls. This outcome is interpreted as supporting the hypothesis, derived from two-stage attention theory, that pups given discrimination training when 16 and 17 days old can solve the discrimination problem when they are 18 days old much faster than task-naive

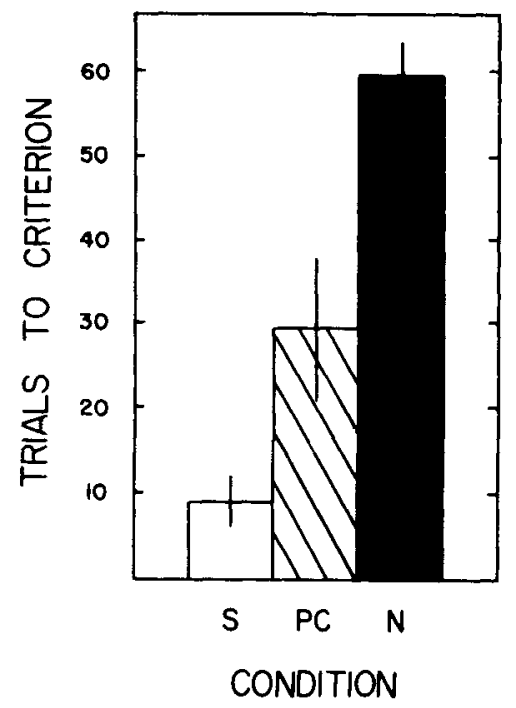

Figure 5. Mean number of trials to criterion during the transfer session of Experiment 3. The data for naive pups is a re-presentation of the data for pups in Experiment 1 that were 18 days old at the start of training on the simultaneous discrimination problem. Key: $\mathrm{S}=$ simultaneous discrimination problem, $\mathbf{P C}=$ procedural control; $\mathbf{N}=$ naive. 
18-day-olds, in part because they had begun to attend to the relevant stimulus dimension (brightness). This finding is important because it provides additional support for the applicability of the two-stage approach to the solving of discriminations by the young rats.

The pups in the procedural control group also benefited substantially from exposure to the general features of the training environment. To make this point clear, the number of trials it took task-naive 18-day-olds from Experiment 1 to solve the simultaneous problem is also included in Figure 5. Procedural-control pups took only about half as many trials to reach criterion as did task-naive 18-dayolds. The performance of the pups in the procedural control condition may have benefited from their having learned that spatial cues were irrelevant to the solution as well as from their having had the opportunity to habituate to the nonspecific aspects of the task.

\section{EXPERIMENT 4}

Given the results of the previous experiments, we suspected that the major difference between the younger and older rats might lie in their attentional as opposed to their associative processes. We also wondered if the attentional deficit could be caused by the young rats' relative inability to ignore irrelevant cues. In the preceding experiments, the irrelevant stimuli that had been most likely to influence the animals' choice behavior were position cues. If the young animals had difficulty in solving the brightness problem because they could not suppress responding to position cues and not because of a general associative deficit, then they should learn a position discrimination with relative ease.

\section{Method}

All subjects $(n=6)$ were 16 days old at the start of the experiment. After pretraining (see Experiment 1 ), the subjects were trained on a position discrimination. On this problem, the platform was always located behind either the left or the right stimulus card. In order to determine its initial position preference, the subject was first given five trials with a platform behind both cards. The side chosen most often on these trials was designated as the subject's preferred side. Training on the position problem then began with the platform located on the subject's nonpreferred side. The subject was then trained until it reached the criterion of 7 of 8 correct choices. To ensure that the pup's position preference was indeed modifiable by training, immediately after this criterion was reached, the platform was moved to the other side and the subject was required to reverse its position preference. The 7-of-8-correct choices criterion was also used for the reversal problem. During both original and reversal training, the black and white stimulus cards were present but were irrelevant to the problem's solution. Just as in the previous experiments, these cards were equally often on the left and right sides. They were alternated according to the procedure described in Experiment 1.

\section{Results and Discussion}

The mean number of trials to reach criterion on the original position problem and its reversal was 2 and 7.8 , respectively. The ease with which 16 -day-olds learned and reversed a position discrimination should be contrasted with their performance on the brightness discrimination problem, with which both 16-and 18-day-olds took over 50 trials to reach criterion (see Figure 2).

This finding is consistent with the hypothesis that the 16-day-olds' problem on the brightness discrimination is attentional rather than associative. It is also consistent with the additional hypothesis that young rats do poorly on brightness discriminations because they have difficulty in learning to ignore or inhibit their responses to irrelevant position cues. If this is true, and young rats have no general associative deficit, then they should be able to readily solve position discriminations. It is unlikely that this problem could be solved in fewer trials than were required by the 16-day-olds in the present experiment. It must be pointed out, however, that these data can also be interpreted to mean that young rats can quickly learn to attend to position cues but have difficulty in identifying brightness cues as being relevant.

\section{GENERAL DISCUSSION}

In the introduction, we noted the possibility that pups might solve the simultaneous brightness problem at an earlier age than they would the successive conditional, and that this would imply that the two tasks were, to some degree, subserved by different processes. Performance on the two problems, however, did not interact with age. At every age, pups required no more training to reach criterion on the successive problem than they did on the simultaneous problem. These data thus provide no evidence that the two tasks are subserved by different processes.

Performance on both the simultaneous and successive brightness discrimination tasks, however, was age dependent. The youngest pups (16- and 18-day-olds) in these experiments required almost six times the number of trials to reach criterion as did the oldest pups (28-day-olds).

Several sources of data converge to indicate that a fundamental difference between the younger and older pups resides in the processes that determine the degree to which they attend to the relevant visual stimuli, as opposed to the associative processes that enable the pups to learn the contingencies correlated with these cues (e.g., $\mathrm{B}+/ \mathrm{W}-$ ).

The results of Experiment 4 rule out the possibility that the youngest animals have a general associative learning deficit. They learned and reversed a spatial position discrimination in remarkably few trials. The data from this experiment also virtually eliminate the possibility that the the 16-18-day-olds' impaired performance on the brightness problem reflects immature motor systems or motivational systems. If they were not able to swim or if they were not motivated to escape the water, then learning a position discrimination should also have been impaired.

The backward learning curves for the different-aged pups (see Figure 3) revealed that the primary difference between young and old pups occurred in the "presolution" period. Younger pups required more trials to "break out" of this stage than did older pups. Pups at every age, 
however, showed equally rapid transitions from the presolution to solution phase. This finding suggests that young pups do not have an associative deficit that is specific to brightness cues.

Additional evidence against the associative deficit account can be derived from Experiment 3. The procedural control animals that were exposed to all aspects of the task except the brightness cues when they were 16-17 days old subsequently solved the brightness discrimination problem (introduced when they were 18 days old) in about 30 trials. This is approximately the same number of trials that task-naive 20-day-olds took to solve the problem (see Figure 2). If the difference observed in Experiment 1 between task-naive 16-18-day-old animals and task-naive 20-day-olds reflected an associative impairment, then the nonspecific training given the 16-17-day-old pups (procedural controls) should not have had any impact on the rate at which they subsequently solved the brightness problem; in fact, however, it made 18-day-olds functionally equivalent to 20-day-olds.

One could also argue that the young rats' impairment on the brightness problem is simply visual, that they cannot discriminate the brightness cues as well as ólder animals. The behavior of the pups in the procedural control condition also argues against the visual deficit interpretation. If the 16-20-day-olds' problem was simply sensory, then it is difficult to see how their exposure to the nonspecific aspects of the task when they were 16-17 days old could make the procedural controls solve the brightness problem in as few trials as 20-day-olds. If one assumes, however, that the 16-20-day-olds' visual system is sufficiently developed to permit them to detect the difference between the black and white discriminanda but that they have an attentional deficit, then one might expect to see that learning brightness discriminations can be facilitated by the treatment that the procedural control pups experience. It is also difficult to see how the visualimpairment interpretation can account for why the rate of transition from the presolution to the solution phase of performance was independent of training age (see Figure 3).

Other evidence consistent with the attentional-deficit interpretation, can also be marshaled. The negative transfer paradigm employed in Experiment 2 provided no evidence that the younger pups (16-17-day-olds) learned the specific contingencies associated with the black and white discriminanda during the so-called presolution period. For example, pups trained for two sessions on a $\mathrm{B}+$ versus $\mathrm{W}-$ simultaneous discrimination reached criterion as fast when they were transferred to a B - versus $\mathrm{W}+$ problem as did pups trained throughout on the $B-$ versus $W+$ problem. If they had acquired the relevant associations in the presolution period, the pups in the transfer condition should have taken more trials to reach criterion. The absence of such specific learning during the presolution period is consistent with the view that the young pups failed to learn about the brightness cues because they were not attending to them.
Even though young pups apparently learned very little during the presolution period about the specific contingencies associated with the brightness cues, the results of Experiment 3 suggest that by the end of the second session of training, they were beginning to attend to these stimuli. Pups trained with the brightness cues relevant when they were 16 and 17 days old required fewer trials to reach criterion than did the procedural control pups.

This review of the data argues against sensory, motor, motivational, or associative (general or specific) deficits as underlying the young rats' impaired performance on the brightness discrimination learning problems. At this time, however, we are unable to reject the hypothesis that there is a fundamental difference between younger and older rats in their attentional processes.

The nature of this attentional difference might lie either (1) in the failure of young animals to attend to brightness cues because they have difficulty in learning to ignore irrelevant cues, such as position cues, or (2) in the existence of a specific deficit in the process that enables the young animals to orient to and process visual stimulation. The former hypothesis is a version of a class of attention theories (Zeaman \& House, 1963; Lovejoy, 1968, Sutherland \& Mackintosh, 1971) that share what Thomas (1970) has called the "inverse" hypothesis. According to this class of theories, stimuli are thought to compete for access to some limited-capacity attentional process such that attention to one stimulus configuration will be at the expense of others. The latter hypothesis is similar to Mackintosh's (1975) suggestion that the attention-capturing properties (termed salience) of different stimulus events are independent, and that attending to one event is not at the expense of attending to another event.

We are currently biased toward favoring the "inverse" version of the attentional hypothesis. This is primarily because the 16-18-day-old procedural control animals of Experiment 3 , which were pretrained to all features of the task except the brightness cues, subsequently learned the brightness discrimination in half as many trials as did similar-aged task-naive animals. Thus, the training given the procedural controls increased their likelihood of attending to the brightness cues (increased their salience). For the salience of a stimulus dimension to increase through learning, an attentional theory that assumes that stimulus dimensions have independent saliences (Mackintosh, 1975) would require that dimension to be correlated with a favorable set of reinforcement contingencies. This condition could not possibly have been met for the procedural control animals. First, the specific black and white brightness values were not present during the pretraining phase. Second, in the pretraining phase, all stimulus dimensions that were present, including brightness, were irrelevant to platform location.

In contrast, the behavior of the procedural controls is easily interpreted by the "inverse" attentional account. During pretraining, the pups learned that some stimulus dimensions (e.g., spatial location) were irrelevant. Thus, the ability of the irrelevant cues to compete for attention 
was reduced, and that of the subsequently introduced brightness cues was correspondingly enhanced.

\section{REFERENCES}

EHRENFREUND, D. (1948). An experimental test of the continuity theory of discrimination learning with pattern vision. Journal of Comparative \& Physiological Psychology, 41, 408-422.

KRECHEVSKY, I. (1932). A study of the continuity of the problem-solving process. Psychological Review, 45, 107-133.

KRECHEVSKY, I. (1938). Antagonistic visual discrimination habits in the white rat. Journal of Comparative Psychology, 14, 263-277.

LASHLEY, K. S. (1930). The mechanisms of vision. I. A method for rapid analysis of pattern-vision in the rat. Journal of Genetic Psychology, 37, 453-460.

LASHLEY, K. S. (1963). Brain mechanisms and intelligence: A quantitative study of injuries to the brain. New York: Dover. (Original work published 1929)

LAWRENCE, D. H. (1949). Acquired distinctiveness of cues: II. Transfer between discriminations on the basis of familiarity with the stimulus. Journal of Experimental Psychology, 39, 175-188.

LoveJoY, E. (1968). Attention in discrimination learning. San Francisco: Holden-Day.

MackINTOSH, N. J. (1965). Selective attention in animal discrimination learning. Psychological Bulletin, 64, 124-150.

Mackintosh, N. J. (1975). A theory of attention: Variation in the associability of stimuli with reinforcement. Psychological Review, 52, 276-295.
Morris, R. G. M. (1981). Spatial localization does not require the presence of local cues. Learning \& Motivation, 12, 239-260.

MoYe, T. B., \& RudY, J. W. (1985). Ontogenesis of learning: VI. Learned and unlearned responses to visual stimulation in the infant hooded rat. Developmental Psychobiology, 18, 395-409.

Rudy, J. W., Stadler-Morris, S., \& Albert, P. (1987). Ontogeny of spatial navigation behaviors in the rat: Dissociation of "proximal" and "distal"-cue-based behaviors. Behavioral Neuroscience, 101, 62-73.

SCHENK, F. (1985). Development of place navigation in rats from weaning to puberty. Behavioral \& Neural Biology, 43, 69-85.

SPENCE, K. W. (1936). The nature of discrimination learning in animals. Psychological Review, 43, 427-449.

SPENCE, K. W. (1945). An experimental test of continuity and noncontinuity theories of discrimination learning. Journal of Experimental Psychology, 35, 253-266.

Sutherland, N. S., Mackintosh, N. J. (1971). Mechanisms of animal discrimination learning. New York: Academic Press.

Thomas, D. R. (1970). Stimulus selection, attention, and related matters. In J. H. Reynierse (Ed.), Current issues in animal learning. Lincoln: University of Nebraska Press.

Zeaman, D., \& House, B. J. (1963). The role of attention in retardate discrimination learning. In N. R. Ellis (Ed.), Handbook of mental deficiency: Psychological theory and research. New York: McGraw-Hill.

(Manuscript received June 9, 1986;

revision accepted for publication December 20, 1986.) 